\title{
Search for sdB/WD pulsators in the Kepler FOV
}

\author{
R. Silvotti, ${ }^{1}$ G. Handler, ${ }^{2}$ S. Schuh, ${ }^{3}$ B. Castanheira, ${ }^{2}$ and H. Kjeldsen ${ }^{4}$ \\ ${ }^{1}$ INAF - Osservatorio Astronomico di Capodimonte, via Moiariello 16, 80131 Napoli, Italy \\ 2 Institut für Astronomie, Universität Wien, Türkenschanzstrasse 17, 1180 Vienna, Austria \\ 3 Institut für Astrophysik, Universität Göttingen, Friedrich-Hund-Platz 1, 37077 Göttingen, Germany \\ ${ }^{4}$ Dept. of Physics and Astronomy, Aarhus University, Ny Munkegade, 8000 Aarhus C, Denmark
}

\begin{abstract}
In this article we present the preliminary results of an observational search for subdwarf $B$ and white dwarf pulsators in the Kepler field of view (FOV), performed using the DOLORES camera attached to the $3.6 \mathrm{~m}$ Telescopio Nazionale Galileo (TNG).
\end{abstract}

Individual Objects: KIC10_05807616, KIC10_02020175

\section{Introduction}

The Kepler satellite will be launched in March 2009 and will observe a 105 square degree field for 4 years with the primary goal of finding new exoplanets using the transit method. Kepler's secondary goal is asteroseismology: the objective is to characterize stars hosting planets, and also to study in detail a few thousands other oscillating stars. Among the seismic targets, up to 512 stars can be observed in short cadence with a sampling time of 1 minute (for all the other targets the cadence will be $30 \mathrm{~min}$ ), allowing the study of short period pulsators, including hot subdwarfs B (sdBs) and white dwarfs (WDs). Thanks to its exceptional photometric accuracy and duty cycle $(\approx 95 \%$, see Christensen-Dalsgaard et al. 2006 for more details), Kepler can produce numerous exciting results on these stars: 1 ) detect low-amplitude $(\lesssim 100 \mathrm{ppm})$ and high-degree $(I>2)$ modes, not visible from the ground. 2) Measure stellar global parameters with unprecedented accuracy (mass, rotation, $\mathrm{H} / \mathrm{He}$ layer thickness, $T_{\text {eff }}, \log g$ ). 3) Improve our understanding of the physics of these stars (differential rotation; core $\mathrm{C} / \mathrm{O}$ ratio and equation of state, neutrino cooling and crystallization in WDs). 4) Study amplitude variations and nonlinear effects. 5) Through the O-C diagram, measure $\dot{P}$, determine the evolutionary status of the star and search for low-mass companions (BDs/planets) with masses down to $\approx 10^{-1} \mathrm{M}_{\text {Jup }}$ (see the recent example of V391 Peg b, Silvotti et al. 2007).

\section{Observations and preliminary results}

The 24 targets were selected from the KIC10 (Kepler Input Catalogue version 10, used internally by the Kepler team to select targets) through their $g-i$ SLOAN colour. For most of the targets proper motions were available from the USNO catalogue allowing to refine the selection using a reduced proper motion diagram.

The time-series photometry was performed during a single run at the $3.6 \mathrm{~m}$ TNG in August 2008. Each target was observed for 1 to 2 hours with the SLOAN g filter, with exposure 


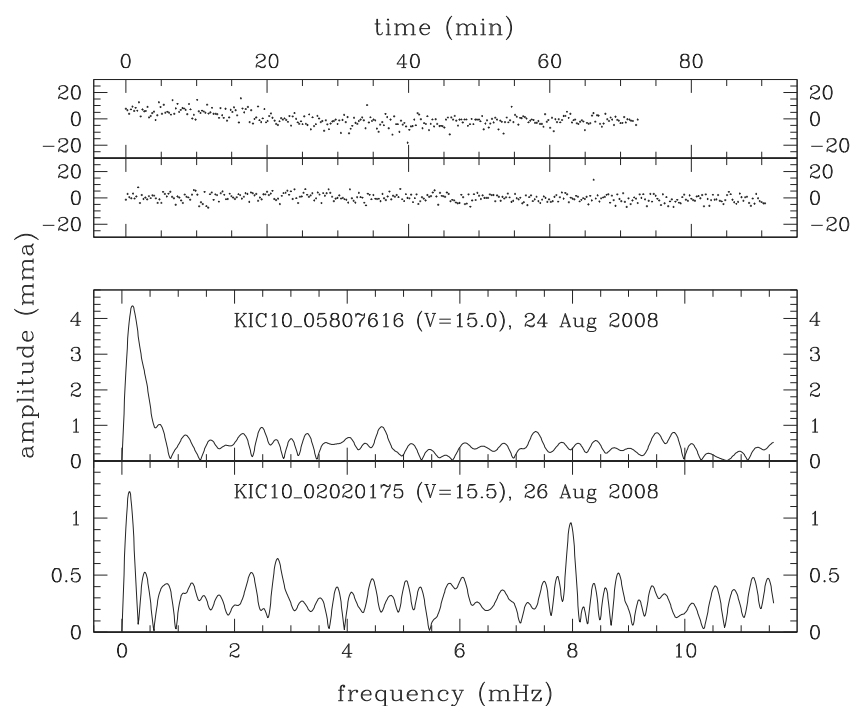

Figure 1: Light curve and amplitude spectrum of the two best pulsator candidates (see text for more details).

times between 1 and $10 \mathrm{~s}$, depending on the magnitude. Details on the selection criteria and data reduction will be given in a forthcoming paper (Silvotti et al., in preparation).

A new variable star was found from a preliminary analysis of our data. It is probably a cataclysmic variable and we will present it in a future article. However, our data did not revealed any star with a clear signature of intrinsic pulsations. The typical upper limits that we have obtained for the pulsation amplitude are between 1 and $2 \mathrm{mma}(1 \mathrm{mma}=$ millimodulation-amplitude $=1000 \mathrm{ppm}$ ). Nevertheless, for two targets having colours compatible with sdB stars, the light curve or the amplitude spectrum suggest possible periodicities. The upper panel target in Fig. 1 shows a periodicity near 1 hour, compatible with a slow (g mode) sdB pulsator. The lower panel target has a peak higher than 3 times the local noise $(\mathrm{S} / \mathrm{N}>3)$ at about $125 \mathrm{~s}$, which would correspond to a rapid ( $\mathrm{p}$ mode) sdB pulsator.

New observations of these two stars done in October 2008, and now under reduction, will help to clarify whether they oscillate or not. Just before submitting this article, we have been informed that all the 24 targets observed at the TNG have been included in a list of survey targets that should be observed by Kepler in the first months of the mission to verify their pulsational stability with a much lower detection threshold.

Acknowledgments. The authors are very grateful to Alfio Bonanno and Silvio Leccia for having given part of their six TNG nights to this programme, following technical problems with the SARG instrument. Silvio Leccia has been also observing during the first night. RS wishes to thank the TNG technical team, in particular Gloria Andreuzzi, Antonio Magazzu and Luca Difabrizio, who did an excellent job during the 7 nights of service-mode observations.

\section{References}

Christensen-Dalsgaard, J., Arentoft, T., Brown, T. M., et al. 2006, CoAst, 150, 350

Silvotti, R., Schuh, S., Janulis, R., et al. 2007, Nature, 449, 189 